\title{
Border Detecting Method for IR Image of Fire Extinguishing Agent Dispersion
}

\author{
Guan xiangyi ${ }^{1,2}$, Liu shaogang ${ }^{2}$, Ma bingpeng ${ }^{3}$, Sun zhenbao ${ }^{3}$, Zhao lili $^{1}$ and Xu ying ${ }^{4}$ \\ ${ }^{1}$ School of Light Industry, Harbin University of Commerce, \\ Harbin, China \\ ${ }^{2}$ College of Mechanical and Electrical Engineering, Harbin Engineering University, \\ Harbin, China \\ ${ }^{3}$ School of computer and information engineering, Harbin University of Commerce, \\ Harbin, China \\ ${ }^{4}$ School of energy and civil engineering, Harbin University of Commence, \\ Harbin, China \\ guanxiangyi@126.com

\section{Abstract}

The temperature difference between fires extinguishing agent dispersion field caused by grenade explosion scatters and background is and fire extinguishing agent dispersion presents obscure configuration. These induce the lowest contrast of the infrared fire extinguishing agent dispersion image and the borders of the fire extinguishing agent dispersion are difficult to be extracted According to the characters of fire extinguishing agent dispersion and infrared image, a border detecting method by dynamic texture and mathematical morphology was proposed. Firstly, the infrared image sequence was presegmented by dynamic texture method, and then the edges in fire extinguishing agent dispersion were extracted by Canny operalon the extracted edges were dilated, labeled and selected by the mathematicalmorphology and the true dispersion borders were extracted. The experimental results show that processing the fire extinguishing agent dispersion images by the methods mentioned above can effectively extract the borders of the fire extinguishing agent dispersion inages and get better results, which can be the foundation of measuring the fire extinguis hing agent disp rsion.

Keywords: fire extingushing agent dispersion; infrared image; border detecting

\section{Introduction}

Crown fire is a burning in the canopy of the forest fire, which is fairly dangerous and difficult to extmguish [1]. Remote drop of fire extinguishing bomb has became an effective method to extinguish crown fire. After dropping the extinguishing bomb, the dispersion area has been affected by the gas flow in the fire ground so that air flow has a major influence over the effectiveness of fire extinguishing agent. Meanwhile, the crowns of foliage conditions also have an impact on the dispersion. Through experimental analysis, the current actual quantity of fire extinguishing bomb used to extinguish crown fire is equivalent to 3-4 times the theoretical amount. The measurement of dispersion area would help us study the interaction between fire extinguishing agent and fire ground for a more effective usage of fire extinguishing agent. Explosive dispersion is hard to measure, most scholars draw FAE fuel explosive dispersal process to analyze the dispersion area. However, the result was not precise according to parameter back from the results. 
This paper will analyze the interaction between fire extinguishing agent and fire ground based on the infrared image that has been development in recent years [2-7]; the dispersion border will be highlighted; the interaction would be analyzed in order to determine the best explosion point [8-9], assure the best result of fire extinguishing.

\section{Extinguishing Agent Dispersed Arrangement of Field Experiments}

The explosion of fire extinguishing bomb to disperse ultrafine powder fire extinguishing agent and the effect on the fire ground have been measured. The fire extinguishing bomb used in the experiment has an outer radius of $255 \mathrm{~mm}$, shell thickness of $3 \mathrm{~mm}$, the shell material of FBR, ultrafine dry powder extinguishing agent of $13 \mathrm{~kg}$ with density of $0.3 \mathrm{~g} / \mathrm{cm}^{3}$. Center tube contains RDX explosive. The extinguishing fire bomb is placed above the center of the fire ground that is simulated as crown fire. Distant record and infrared thermal imager have also been placed. Seen figure below:

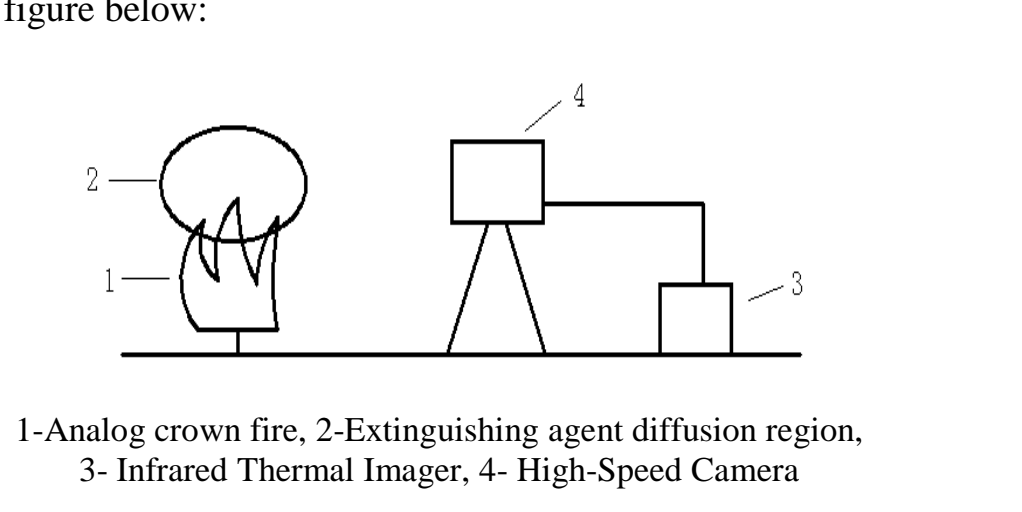

Figure 1. Experimental System Layout

The extinguishing process has been recorded by the infrared imager. The early, mid, late and the end of dispersion have been chosen to analyze the interaction between fire extinguishing bomb and fire ground see Figure 2. Those pictures have been divided into two parts, which are fire extinguishing agent dispersion area and background. The borders are not very clear that the transition also exists.

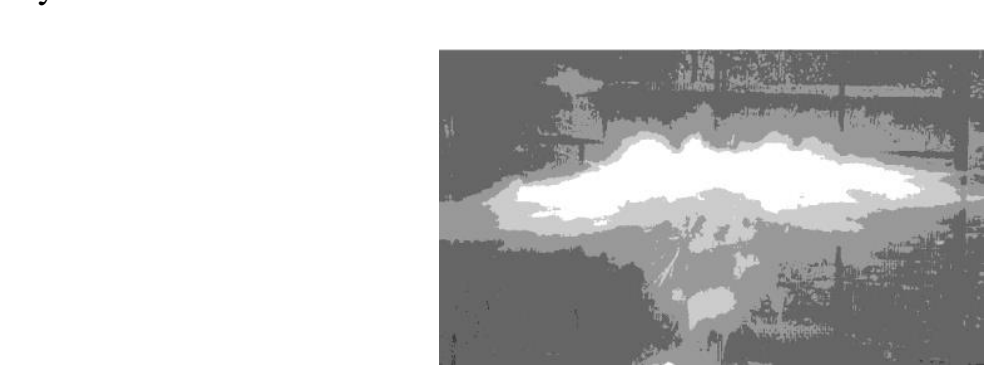

Figure 2. Extinguishing Agent Dispersion Infrared Image

\section{Extinguishing Agent Dispersed Field of Dynamic Infrared Image Texture Segmentation}

The dynamic texture is texture image sequences which refers to a repetitive spatial and correlation on time. For example, the river flows, flame, smoke dispersion with wind and so on [10-13]. Each frame is static texture, there is a certain statistical properties of the time frame between the front and rear. The fire extinguishing agent dispersion is a typical kind of 
dynamic texture, the production, diffusion, dissipation and other processes of dynamic texture is significantly different from the surrounding environment backgrounds. Diffuse infrared image sequences by infrared thermal imager extinguishing agent can be recorded this dynamic texture segmentation. It can be divided into three categories: (1) the method was based on model; (2) the method was based on the motion; (3)the method was based on a special. This paper uses a dynamic texture segmentation method based on Fourier analysis of the proposed by LI. The segmentation process was shown in Figure 3.

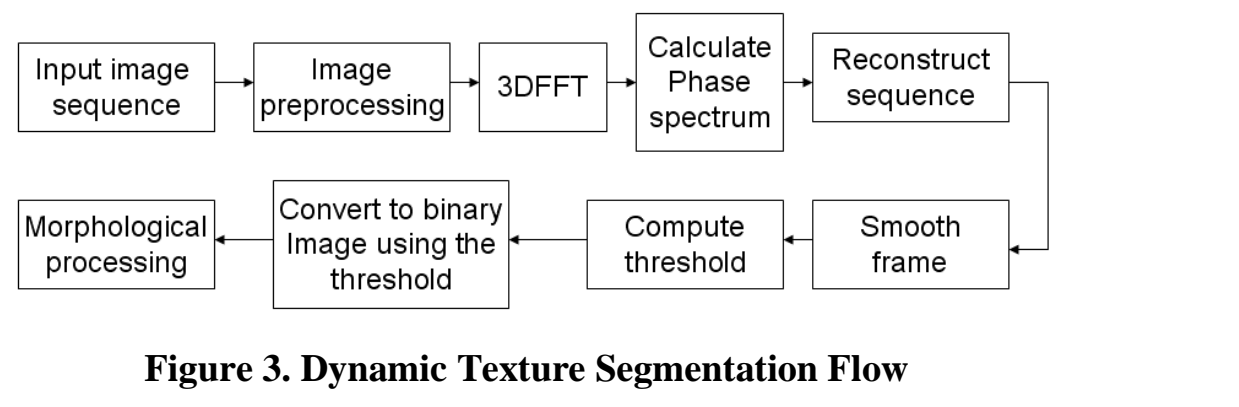

In the pre-processing stage, the traditional contrast enhancement, histogram equalization and other methods of infrared image enhancement was used [14-16. Infrared grayscale image sequence $I(x, y, t)$, Wherein $x, y$ is the position of each pixerin the representative; $t$ is the frame number. For $\mathrm{I}(\mathrm{x}, \mathrm{y}, \mathrm{t})$ for $3 \mathrm{D}$ discrete Fourier transform (DFT) can be expressed as:

$F(I(x, y, t))=\sum_{x=1}^{x-1}\left(\omega_{x}^{k_{1 x}} \sum_{y=0}^{y-1}\left(\omega_{Y}^{k_{2 y} y} \sum_{x=0}^{t-1} \omega_{T}^{k} I(R, y, t)\right)\right.$

Wherein: $\mathrm{x}$ and $\mathrm{y}$ are the width and height of ach frame; $\mathrm{t}$ is the total number of frames. $\omega_{x}=\exp { }^{(-2 \pi i / x)}, \quad k_{1}=0, x-1$. After the image sequence $\mathrm{I}(\mathrm{x}, \mathrm{y}, \mathrm{t})$ during the $3 \mathrm{D}$ Discrete Fourier Transform (DFT), By $F(\mathrm{H}(\mathrm{x}, \mathrm{y}, \mathrm{t}))$ of the real part and the imaginary part to obtain a phase spectrum $P$ [F $(\mathbb{I}(x, y, t))]$, 3D phase inverse DFT spectrum, $\hat{I}(x, y, t)=\mid e^{-1}\left\{\left.[F(I(x, y, t))]\right|^{2}\right.$

When performing 3D discrete Fourier transform (DFT), the number of frames must be selected considering the frame rate of the image sequence, and agent dispersion rate of change. Each frane reconstruction filters smoothes the average sequence. Choose a suitable threshold (such as mean) gray value of the image sequence into two image sequences. Binary image obtained by mathematical morphology operations, including filling, erosion, dilation, opening and closing operation. You can use the morphological opening operation to operate filter isolated point arithmetic expansion before proceeding. When performing the role opposite-morphological operations, such as corrosion, expansion, structural elements of the type and size used should be consistent.

Figure 4 shows the dynamic texture segmentation through image after. Clearly, because of the dispersion and the surrounding area extinguishing agent unperturbed background texture characteristic differences in the dynamic texture segmentation can more accurately determine the area where the fire extinguishing agent dispersed. 


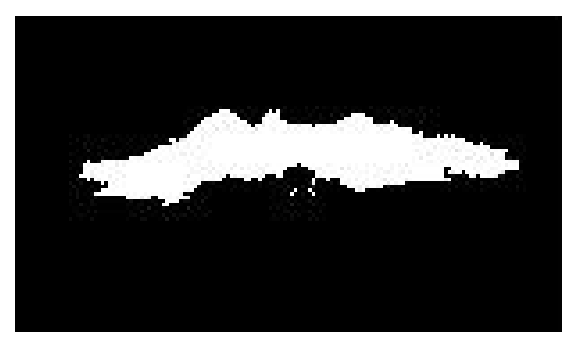

Figure 4. Segmented Image

Through the use of multiple image sequences dynamic texture segmentation method found that due to the low frame rate selected infrared image sequences and morphological processing elements in the shape of the structure is limited, segmented extinguishing agent dispersion area is not complete, but very accurate circle the location where the wake region. Thus you can use this method of extinguishing agents dispersed areas pre-segmentation, followed by other operations. Figure 3 shows the outside of the dynamic texture segmentation method based on the resulting image extinguishing agent dispersed area, fire extinguishing agent diffusion zone part has been removed.

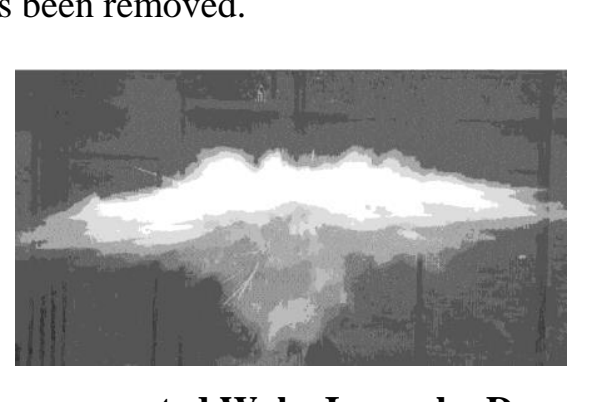

Figure 5. Pre-segmented Wake Image by Dynamic Texture

\section{Edge Detection}

Figure 5 is divided into the image field of fire-extinguishing agent dispersed in two parts and the background, the boundary between these two areas is not particularly clear, there is a mutual transfer of the transition zone. In order to facilitate the analysis of the precise scope of extinguishing agent dispersed field and background. Already largely determine extinguishing agents diffuse field edge extraction operation, commonly used methods are: Prewitt, Canny, Bwperim, Laplacian, R6bert, Sobel operator, morphological operators and other methods [1718]. Operator using different methods such as edge extraction after different. The experimental comparison, we chose the Canny operator, the operator of the extinguishing agent to extractmore complete dispersion field.

Figure 6 shows the use of Canny operator from extinguishing agents diffuse edges of the image field.

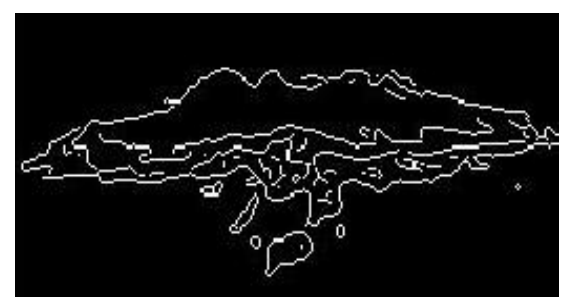

Figure 6. Boundary of Extinguishing Agent Diffusion Field Detection 
After the border has been detected, the images were also recovered. Images were divided into two levels of black and white by the method of threshold. Q is the gravity value of the histogram. The background of the images was also one of two levels. The gravity value of black and white areas can be calculated by histogram.

$$
A_{w}=\frac{\sum_{i=Q}^{n} g_{i} q_{i}}{\sum_{i=Q}^{n} q_{i}}, A_{b}=\frac{\sum_{i=0}^{Q} g_{i} q_{i}}{\sum_{i=0}^{Q} q_{i}}
$$

Purpose of the calculations of $A_{w}$ and $A_{b}$ is the standard method to find the image restoration and to reduce the diffusion of histogram. After measuring the background image, $A_{w}$ and $A_{b}$ can be gathered in the histogram. Calculations can be completed through the following mapping function, and the image would be transferred from the spatial domain to the blurred domain [7-8].

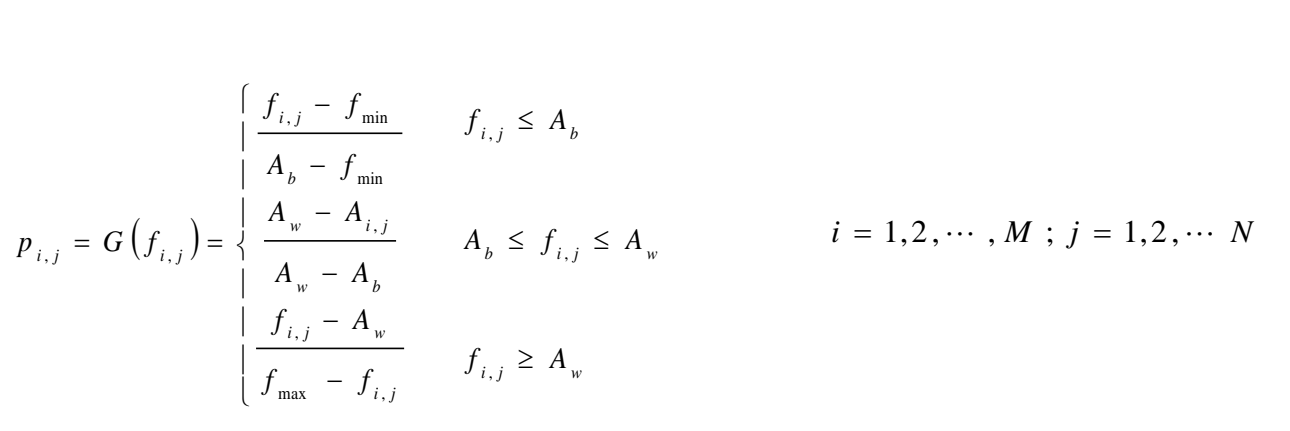

When all the pixels in the gray scale mapped to the interval between 0 and 1 , We apply the transfer function $\mathrm{H}$ on pixels.

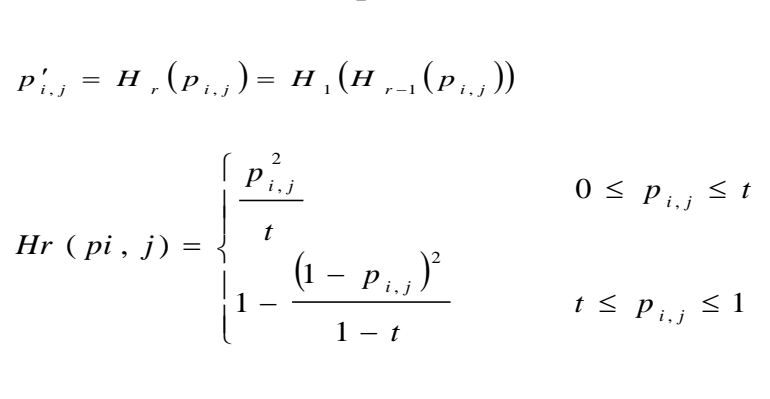

The images were processed by the method offered by literature 2, the histogram could be built. According to the characteristics of the image histogram threshold was adjusted 218, Diffusion pegion and fire area binary image was given, as shown in Figure 4.

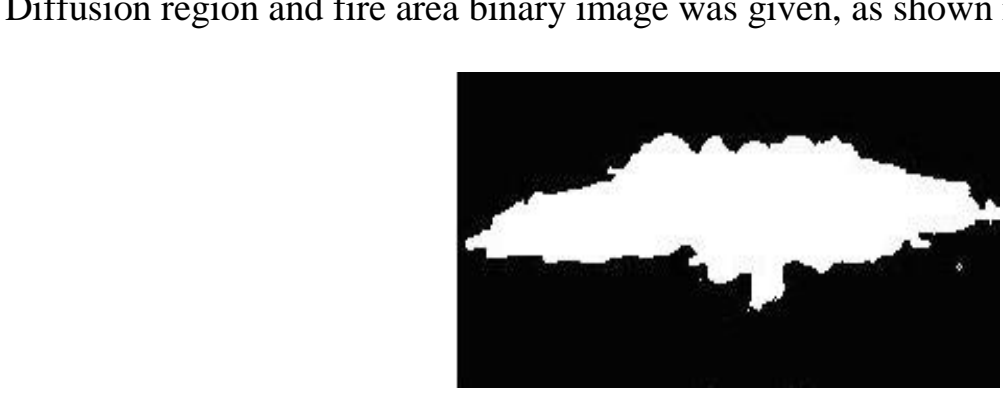

Figure 7. Histogram and Binary Image 
After the process introduced above, the border of fire ground and the area of explosion can be clearly detected, and the background area was changed into binary image. Area calculation application calculated different areas of explosions. The statistics table gives the ratio of fire ground area to the whole area of the image. When the position of camera and the size of picture were fixed, the fire area can be compared horizontally [9-10].

\section{Extinguishing Agent Diffusion Process Analysis}

In the early stage of fire extinguishing agent dispersion, the force of explosion determined the dispersion state. Explosive force depends on the size of the center air powder and natural and strength of shell. Fire extinguishing agent along the direction of explosion air flow accelerated motion. In the acceleration process, the gravity and the force of medium were acted on the fire extinguishing agent particles. The second stage of dispersion, the force of the explosion reduced gradually and equaled to the resistance force acting on the particles. At this time, the speed of fire extinguishing agent reached maximum. Next step, the yelocity of extinguishing agent particle gradually decreased. When the speed is zero, the radius of diffusion arrive maximum. In the end, fire extinguishing agent tu bulence.

According to the dispersion process, the maximum radius of the diffuse infrared image was shown in Figure 8. We also use dynamic texture segmentation and canny algorithm for image processing, so we can get diffusion radius and volume Figure 9 shows the infrared image of the turbulence. We use matlab software to calculate the diffusion zone area, specific data seen in Table 1.

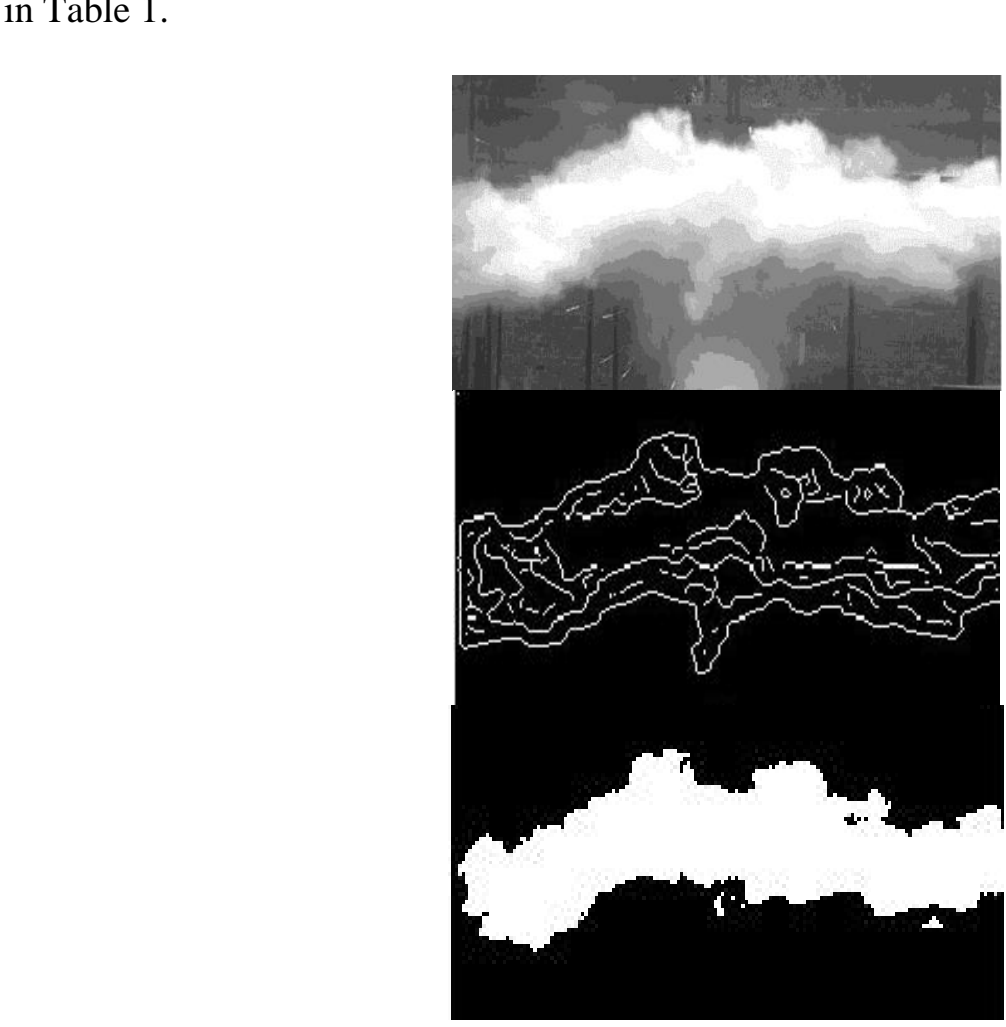

Figure 8. The Maximum Radius of Diffusion Images 


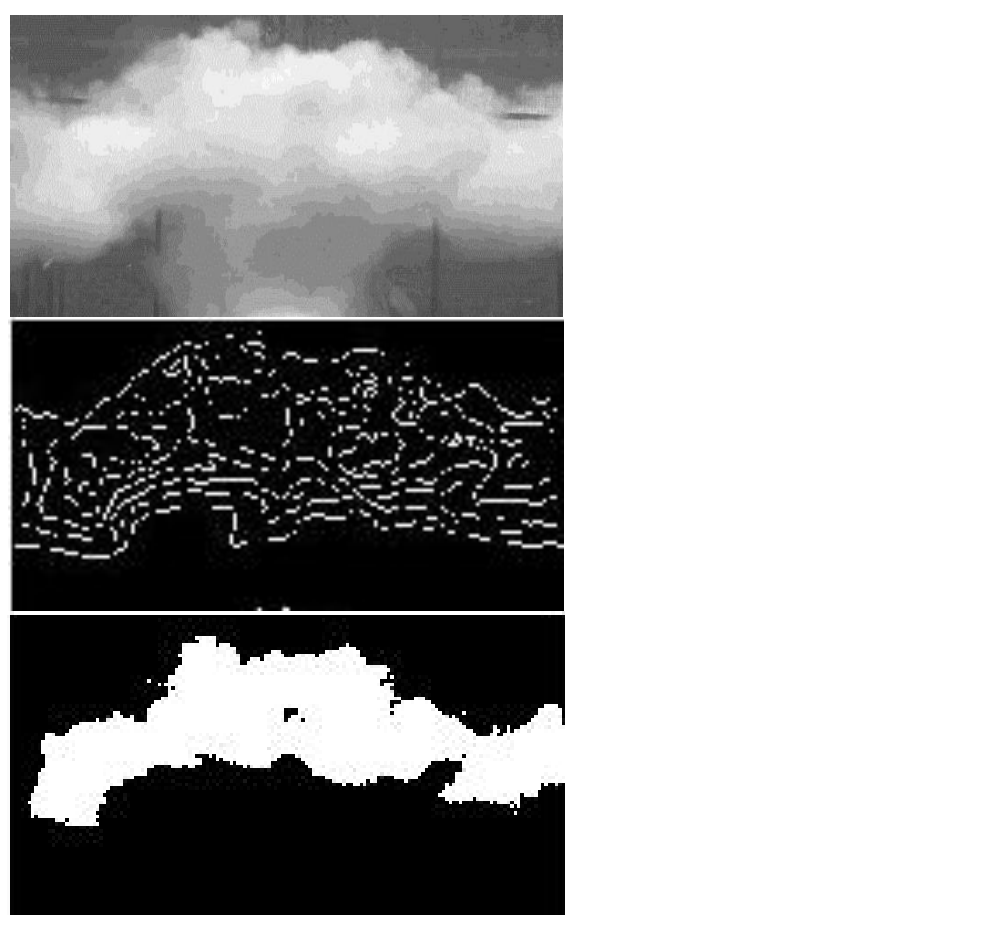

Figure 9. Turbulent Diffusion Image

Table 1. Area of Diffuision Region

\begin{tabular}{|c|c|}
\hline Initial diffusion & Turbulent diffusion \\
\hline 1.49 & 1.92 \\
\hline
\end{tabular}

According to Table 1 With the increase of dispersion area, the dispersion area of the beginning differs greatly from that of the ultimate range with the little effect of turbulent phase on dispersion area. Fire extinguishing mainly concentrates in the process of dispersion area increase. In the dispersion areas of Figure 8 and 9, dispersion concentration can be calculated based on thermalymaging distance and angel of inclination to infer if the dispersion concentration reaches the minimum required by fire extinguishing. The result is the reference for deteching effectiveness of fire extinguishing.

\section{Conclusion}

Aimed at the-low contrast of infrared imagery when fire extinguisher dispersing, an edge detection method based on Canny operator and dynamic textures is employed in this paper. Becaus of the significant difference between dispersion region texture and background, dynamic textures method based on Fourier transform is used for pre-segmentation of dispersion area and detecting the edges. Infrared imagery can be well treated by this method. What's more, it provides the strong guarantee for detecting the effectiveness of fire extinguishing and optimizing the design of the fire extinguishing bomb. After the fire extinguishing bomb test, the ratio of bomb height to diameter was 3.3, the ration of explosion power to fire agent was $1.5 \%$. Fire extinguishing bomb can extinguish crown fire, the amount of fire extinguishing agent reduces $20-25 \%$. For the future improvement of the different types of crown fire extinguishing bombs structural foundation, provide some technical support. 


\section{Acknowledgement}

Natural Science Foundation for young teachers of Harbin University of Commerce (HCUL2013003) Fund for the doctoral program of higher school (20102304110007)

\section{References}

[1] E. Pastor, L. Zárate , E. Planas and J. Arnaldos, Progress in Energy and Combustion Science, vol. 2, (2003), pp. 29.

[2] S. Yanfeng, S. Xiaopeng and X. Jun, Infrared and Laser Engineering, vol. 2, (2008), pp. 37

[3] Y. Tianhe and D. Jingmin, Infrared and Laser Engineering, vol. 1, (2010), pp. 39.

[4] J. Weiqi, L. Bin and F. Yongjie, Infrared and Laser Engineering, vol. 12, (2011), pp. 40.

[5] L. Ai-min, Z. Qi and L. J.-P., Transactions of Beijing Institute of Technology, vol. 2, (2005), pp. 25.

[6] W. Lei, Z. Wenjing and Z. Tiedong, Infrared and Laser Engineering, vol. 14, (2013), pp. 42.

[7] Z. Shiping, X. Xi and Z. Qingrong, Journal of Optoelectronics. Laser, vol. 10, no. 19, (2008).

[8] C. Xudong, Z. Yong and M. Peng, Journal of Combustion Science and Technology, vol. 6, (2012), pp. 18.

[9] H. Mingjun, Z. Tao and G. Xueyong, Chinese Journal of High Pressure Physics, vol. 2, (2004),pp, 18.

[10] W. Bing jian, L. Shang Qian, Z. Hui xin and L. I. Qing, Acta Photonica Sinica, vol. 2, (2005), pp. 34

[11] Q. Yi, L. Huanying and C. Shengtao, Pacific Rim Conference of Lâsers and Electro-optics, (2009).

[12] C. Jie, S. Jiyin and C. Biao, Infrared and Laser Engineering, vol. 6-(2007), pp. 36.

[13] M. O. Szummer, "Temporal Texture Modeling”, Massachusetts 1nstitute of Technology, (1995).

[14] T. Amiaz, S. Fazekas and D. Chetverikov, Proceedings of the st International Conference on Scale Space and Variational Methods in Computer Vision, (2007), pp.

[15] K. Otsuka, T. Horikoshi and Sl. Suzuki, Proceedings of the 14th International Conference on Pattern Recognition, (1998), pp. 1047-1051.

[16] L. Jianghong, C. Liang and C. Yuanhu, Moden Applied Science, vol. 3 (2009), pp. 9.

[17] Z. Hongqun, Z. Xue and X. Wangxin, Infrared and Laser Engineering, vol. 1, (2003), pp. 32.

[18] Y. Yang, W. Jingru and C. Changbin, Infrared and Laser Engineering, vol. 5, (2004), pp. 33.

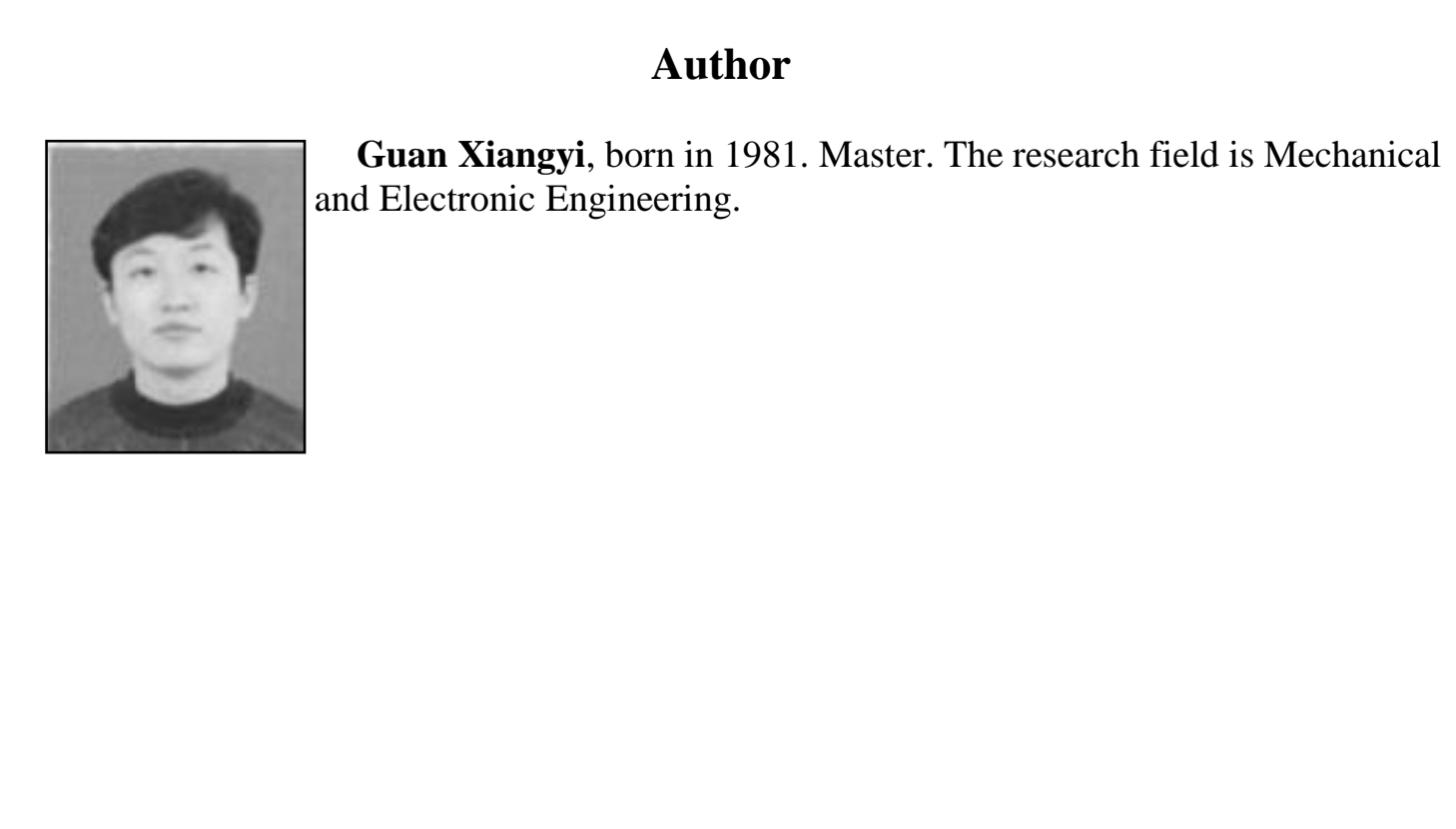

